\title{
Unusual Skin Metastasis from Gastric Cancer: A Case Report
}

\author{
Vicky Sumarki Budipramana \\ Digestive Surgery Division, Department of Surgery, Faculty of Medicine, Universitas Airlangga/Dr. \\ Soetomo General Academic Teaching Hospital Surabaya, Indonesia
}

\begin{abstract}
Background: Skin metastasis from gastric cancer are relatively rare, but they have important clinical significance because they usually indicate a worse prognosis. Skin metastasis from gastric cancer may spread through lymphatic ducts, blood vessels in the ligamentum teres, contiguous extension, or embryologic remnants of the umbilicus. Purpose: To report an unusual skin metastasis from gastric cancer that clinically resembles as a benign lesion of skin papules. Case: A rare metastasis from gastric cancer origin in a 61-year-old male in the abdominal skin. Gastric cancer metastasis in an unusual site such as the abdominal skin often resembles as dermatitis, especially in the early course of metastatic spreading, which looked like common papules on the skin. Discussion: In the beginning, the skin papules were not suspected to be a metastatic process because there was no palpable or no tenderness in the subcutan or fascia underneath. The appearance resembled common dermatitis. However, as papules rapidly spread to the surrounding skin, the malignancy was then suspected. The incisional biopsy from the papules was performed and showed that it was metastatic diffuse-type gastric adenocarcinoma. Conclusion: The skin metastasis was different from general nodules, but rather as skin papules of general dermatitis in initial appearance. The surgeon and dermatologist should be alert if they find any benign appearance of a skin lesion on the abdominal wall in a patient with a history of gastric cancer surgery.
\end{abstract}

Keywords: Gastric cancer, gaster carcinoma, skin metastasis.

Correspondence address: Vicky Sumarki Budipramana, Department of Surgery, Faculty of Medicine, Universitas Airlangga / Dr. Soetomo General Academic Hospital, Jl. Mayjen Prof. Dr. Moestopo No. 6-8, 60131, Surabaya, Indonesia. Phone number : +6231-5501609, e-mail: vickysbudi@gmail.com.

\section{BACKGROUND}

Metastasis of post-resection gastric cancer is frequent; however, the epidemiology of metastatic gastric cancer is unexplored because the cancer registries seldom cover data about metastatic involvement. ${ }^{1}$ Here, we reported a rare case of skin metastasis of gastric carcinoma, clinically presented not as skin nodules but rather multiple abdominal skin papules on the periumbilical region. This case is reported in consideration of its rarity of skin metastasis of gastric cancer to the unusual site with unusual clinical presentation. Skin metastasis is an important sigh of poor prognostic in patients with gastric cancer. ${ }^{2}$

\section{CASE REPORTS}

A 61-year-old male was admitted with a skin lesion on the abdominal wall started to appear 4 months before. The lesion spread to the surrounding skin, and there was a painful sensation in the vertebral area. Physical examination showed multiple reddish skin papules with no pain sensation, and the papules multiplied to the surrounding skin with reddish color on the skin among the papules (Figure 1). The papules were hard on palpation and did not cause any pain. It initially looked like common papules on the skin. However, as it rapidly spread over the surrounding skin, malignancy was suspected. The incisional biopsy from the papules was performed, and the result showed metastatic diffuse-type gastric adenocarcinoma. The patient's medical history revealed that he underwent gastric cancer surgery, and the cancer was found, according to the tumor, node, and metastasis staging system (TNM) was T4N2M0 infiltrating to the tail of the pancreas. The nodes were found in the hilus of the spleen, as shown in the computed tomography (CT) scan (Figure 2).

The total gastrectomy, partial pancreatectomy and splenectomy had been performed one year before. After the surgery, the patient completely recovered. The chemotherapy has been completed. However, eight months after the surgery, a few papules emerged on the abdominal skin around the umbilicus, rapidly multiplied circumferentially to the surrounding skin, and no palpable mass in the abdomen. The abdomen CT scan with intravenous (IV) contrast showed that there were multiple nonspecific lymphadenopathies on the upper left abdomen. The mass located in peripancreatic remnant measured $3.7 \mathrm{~cm}$ x $5.5 \mathrm{~cm}$ x $4.8 \mathrm{~cm}$, bilateral hydronephrosis, and metastatic lesion in the vertebrae as multiple lytic lesions in the corpus and pedicle from lumbal (L)1 to sacrum (S)1. The metastatic masses in the abdomen were unresectable, and the patient suffered from painful sensation over the vertebrae. No definitive treatment was given for the abdominal mass, vertebrae 
lytic lesion, and skin metastasis. The only treatment analgetics. The patient passed away 3 months later. suitable was palliation as supportive care and

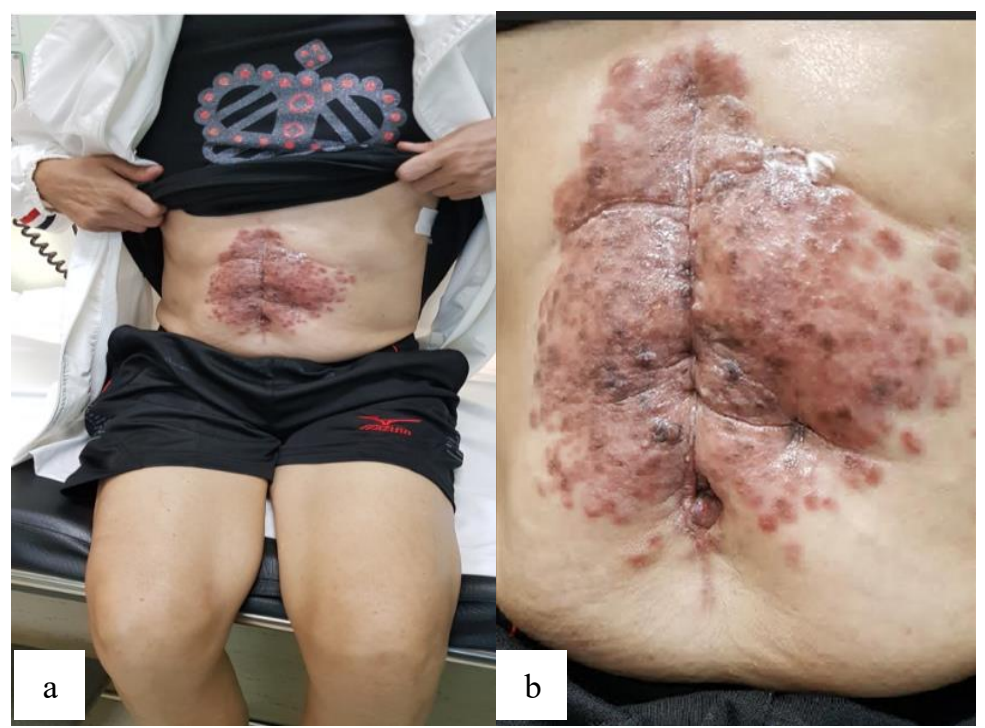

Figure 1. (a,b) Multiple skin papules on the abdominal skin with reddish color on the skin area among the papules.

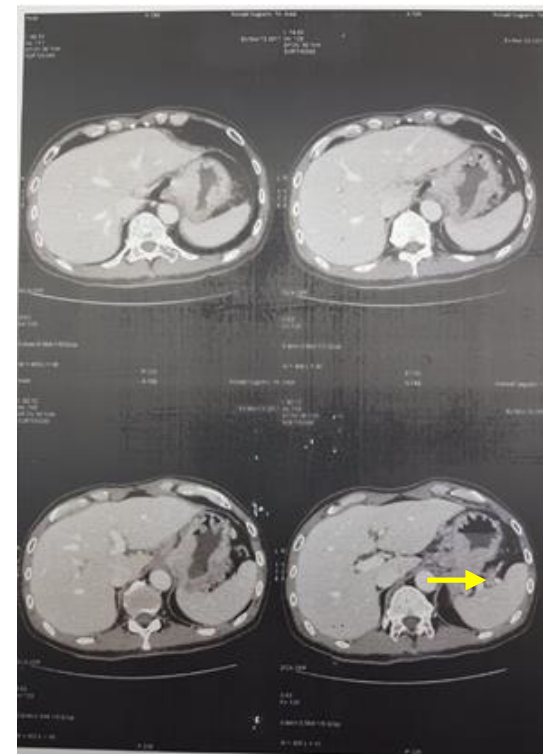

Figure 2. Preoperative computed tomography $(\mathrm{CT})$ scan revealed: gastric cancer infiltration to hilus of the spleen and perigastric nodes (arrow).

\section{DISCUSSION}

Adenocarcinoma metastasis to the skin is uncommon. The incidence of adenocarcinoma from various abdominal organs to the skin is $10.4 \%$, while cutaneous metastasisarising from gastric adenocarcinoma are rare, developing in fewer than 5\% of patients. ${ }^{3}$ In this case, skin lesions were the initial manifestation of the metastatic process, bringing the as skin nodules or Sister Mary Joseph's nodules around patient to the hospital. Initially, the abdominal skin the umbilicus (Figure 3). ${ }^{4,5}$ However, in this case, the metastasis was in the form of papules sized $2 \mathrm{~mm}$ in skin metastasis did not manifest as nodules but as skin diameter located around the umbilicus, with no papules, which initially appeared as dermatitis in general. tenderness palpated in the subcutan underneath, and the Thus, the patient was prescribed with dermal cream appearance was like dermatitis in general. However, treatment. As Sister Mary Joseph's nodules, the papules 
skin metastasis may spread through lymphatic ducts, tumorigenesis and metastasis. Metastatic cells may blood vessels in the teres ligament, contiguous survive more easily if the secondary site has similar extension, or embryologic remnants of the umbilicus. ${ }^{6}$

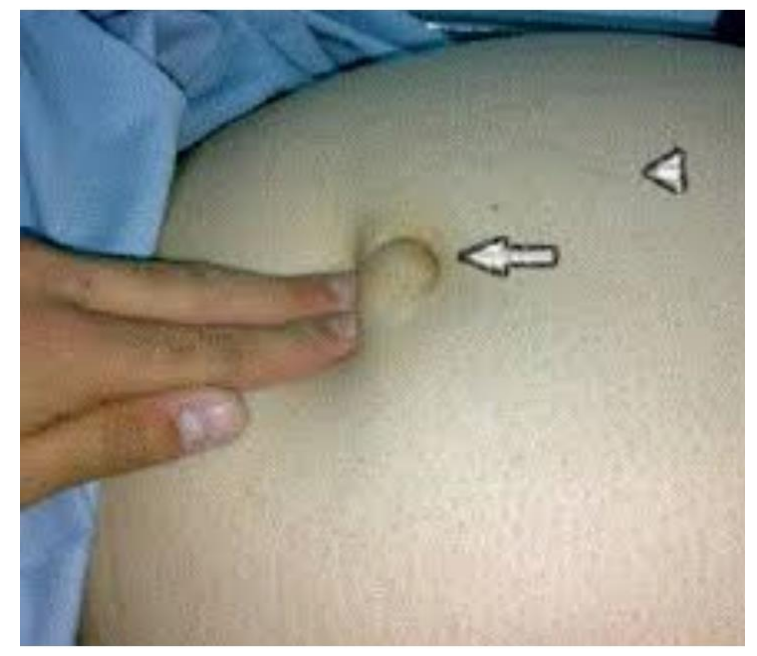

Figure 3. Sister Mary Joseph's nodule in the umbilical area. $^{7}$

In the previous year, the patient was diagnosed with gastric cancer with infiltration to the tail of the pancreas, hilus of the spleen, and peripancreatic nodes (T4bN2M0). The biopsy from gastric mucosa taken at the time of gastroscopy revealed an intestinal-type gastric adenocarcinoma. The surgery performed was total gastrectomy, resection of the tail of the pancreas, splenectomy, and lymph nodes dissection. After the surgery, the patient uneventfully recovered, and chemotherapy Capecitabin + Oxaliplatin was begun 2 months after surgery. The patient completed the chemotherapy according to the regimented schedule. The condition of the patient was good. Eight months after surgery, the patient was aware of a skin papules appearing on the abdominal skin that he returned to the hospital. Initially, the skin papules were not suspected to be a metastatic process because there was no palpable or no tenderness in the subcutaneous or fascia underneath. The appearance was similar to common dermatitis. However, as the papules rapidly spread to the surrounding skin, a malignancy was then suspected. The incisional biopsy from the papules was performed, and the result showed metastatic diffuse-type gastric adenocarcinoma.

The mechanism that predisposes some internal malignancies to metastasize to the skin is not well known. ${ }^{8}$ The skin may provide a favorable environment for the colonization and survival of only certain types of cancer cells. The interaction between tumor cells and certain factors secreted from the dermis or epidermis may play a crucial role in the skin-homing mechanism of metastatic cells. ${ }^{9}$ Recently chemokines and their receptors have shown involvement in characteristics to the primary organ. ${ }^{3}$ The frequency of cutaneous metastasis from carcinomas of the upper gastrointestinal tract in Western countries is lower than $1 \%{ }^{10}$ Gastric adenocarcinoma accounts for the great majority $(95 \%)$ of gastric cancer. Around $9 \%$ of all gastric carcinomas are signet-ring cell adenocarcinoma. Gastric adenocarcinoma usually metastasizes to the liver, peritoneal cavity, and regional lymph nodes than to the skin. ${ }^{11}$ Metastasisof gastric adenocarcinoma account for $5-7 \%$ of all skin metastasiswith much higher frequency, which is $20 \%$, in Japan. ${ }^{3}$ That they are more prevalent in males reflects a higher prevalence of the corresponding primary in males. Metastasisusually appear several months or even years after the primary diagnosis but may also be the presenting sign of the neoplastic process or the presenting sign of the recurrence of the primary tumor. ${ }^{12}$ Cutaneous metastasisof gastric adenocarcinoma usually develop on the abdominal skin, including the umbilicus as the Sister Mary Joseph's nodule, less common sites including the breast, eyelids, back, face, scalp, neck, upper extremities, chest, shoulders/arms, and perianal region. ${ }^{7}$ Such metastasisusually manifest as solitary or multiple red rashes or cutaneous or subcutaneous painless nodules, and are occasionally arranged in a zosteriform distribution; they may also appear as cellulitis or erysipelas-like erythematous plaques (carcinoma erysipelatoid), scarring alopecia or cauliflower-like growths and ulcers, or they mimicked as allergic contact dermatitis on the face. Cutaneous lesions are usually clearly identifiable as metastasisbefore the pathological diagnosis if the patient has a known history of cancer; however, the lesions may be misdiagnosed as benign lesions, such as epidermal cysts, pyogenic granulomas, hemangiomas, herpes zoster or neurofibromas. ${ }^{3}$ In this case, the clinical appearance of metastasis was also unusual in regards to its shape as multiple papules with erythematous skin, contrary to the more usual appearance of one or few nodules such as Sister Mary Joseph's. From a pathologic point of view, the cutaneous metastasis was a diffuse type adenocarcinoma gaster. The skin lesion diagnosis was challenging in those cases. Immunohistochemistry is a useful adjunct in pathological diagnosis. Common pitfalls for pathologists often involve a confusion of staining patterns with the native cutaneous adnexa and distinguishing with the primary adnexal malignancies. ${ }^{13}$ In general, cutaneous metastasis from gastric cancer is considered to be an end-stage presentation of gastric cancer with a poor prognosis. ${ }^{14}$ However, if the metastasis is resectable, and in the absence of metastasiselsewhere, surgical resection could be performed as a treatment option. In this case, resection of 
the skin together with all thickness of the abdominal wall was not a viable option because cancer had already metastasized to the vertebrae.

Diffuse gastric cancer (linitis plastica) has long been recognized as familial, which was not until the recent years that they were discovered to be associated with mutations of specific genes CDH-1. The genes encoding E-cadherin are the most common germline mutations detected in hereditary diffuse gastric cancer (HDGC) syndrome. ${ }^{15}$ However, in this case, we did not find any member of the family of the patient having the same disease or disease related to it. The skin metastasis rapidly spread circumferentially may be caused by the high penetrance behavior of the tumor cells due to the lack of the E-cadherin function. Ecadherin is found within the membrane surrounding the epithelial cells, which are the cells lining the surfaces and cavities of the body and which function to help neighboring cells sticking to one another (cell adhesion) form organized tissues. ${ }^{15}$

\section{CONCLUSION}

We reported a rare case of skin papules in the umbilical area from metastasis of diffuse type of gastric adenocarcinoma. The papules circumferentially and rapidly multiplied to the surrounding skin. This case was reported in consideration of its rarity of skin metastasis from a diffuse type of gastric cancer to the unusual site with unusual clinical presentation. In this case, the skin metastasis was different from generally observed nodules. The skin papules initially looked like general dermatitis. Skin metastasis is an important sigh of poor prognostic in patients with gastric cancer. The surgeon and dermatologist should be alert if they find any benign appearance of a skin lesion on the abdominal wall in the patient with a history of gastric cancer surgery.

\section{REFERENCES}

1. Riihimäki M, Hemminki A, Sundquist $\mathrm{K}$, Sundquist J, Hemminki K. Metastatic spread in patients with gastric cancer. Oncotarget 2016; 7(32): 52307-16.

2. Raka M, Bazzi N, Haidar A, El Safadi A, Chahine G. Metachronous Skin Metastasis of Gastric Cancer: Case Report. JOJ Case Stud 2019; 10(4): 555793

3. Avgerinou G, Flessas I, Hatziolou E, et al. Cutaneous metastasis of signet-ring gastric adenocarcinoma to the breast with unusual clinicopathological features. Anticancer Res 2011; 31(6): 2373-78.
4. Abbasi F, Abbas A, Mahmodlou R, Mehdipour E. Cutaneous metastasis of gastric carcinoma: A rare case with unusual presentation site. Indian $\mathrm{J}$ Dermatopathol Diagn Dermatol 2018; 5:54-6.

5. Liu F, Yan WL, Liu H, Zhang $M$, Sang $H$. Cutaneous metastasisfrom gastric adenocarcinoma 15 years after curative gastrectomy. An Bras Dermatol 2015; 90(3 Suppl 1): 46-50.

6. Piura B. Umbilical metastasis: Sister Mary Joseph's nodule. Harefuah 2006; 145(7): 505-550.

7. Al Ani AH, Totah E, Al Ani A. Sister Joseph Nodule the Durable Sign. General Med 2015; 3(2): 178.

8. Rita V, Rahul K. Kota N, Diwan, Nidhi B. Jivani, and Shailee S. Skin: A mirror of internal malignancy Indian J Med Paediatr Oncol. 2019; 37(4): 214-22.

9. Manuela C, Michele M, Valeria B, Chiara B, Roberta S, Gabriele D, Andrea W, Corrado C, Franco D. Cutaneous Metastasis From Primary Gastric Cancer: A Case Report and Review of the Literature. Cutis 2014; 93: E9-E13.

10. Lookingbill DP, Spangler N, Helm KF. Cutaneous metastasisin patients with metastatic carcinoma: a retrospective study of 4020 patients. J Am Acad Dermatol 1993; 29(2 Pt 1): 228-36.

11. Kim D, Park Y, Joo J, Ryu S, Kim Y, Kim S and Lee J. Clinicopathological characteristics of signet ring cell carcinoma of the stomach. ANZ J Surg 2004; 74: 1060-64.

12. Sánchez-Muñoz A, Gravalos Castro C, Colomer Ryota K, Yoshiaki M, Nozomi M, Toshiki S, Tomonori H. Late Cutaneous Metastasis Originating from Gastric Cancer with Synchronous Metastasis. Case Rep Gastroenterol 2019; 13: 95101.

13. Luo W, Fedda F, Lynch P, Tan D. CDH1 Gene and Hereditary Diffuse Gastric Cancer Syndrome: Molecular and Histological Alterations and Implications for Diagnosis And Treatment. Front Pharmacol 2018; 9: 1421.

14. Ryota K, Yoshiaki M, Nozomi M, Toshiki S, Tomonori H. Late Cutaneous Metastasis Originating from Gastric Cancer with Synchronous Metastasis. Case Rep Gastroenterol 2019; 13: 95101.

15. Luo W, Fedda F, Lynch P, Tan D. CDH1 Gene and Hereditary Diffuse Gastric Cancer Syndrome: Molecular and Histological Alterations and Implications for Diagnosis And Treatment. Front Pharmacol 2018; 9: 1421. 